
\title{
25 Research Soure \\ Multiple hepatoid gastric adenocarcinoma: A case report and literature review
}

\section{Ce Zhu}

Wenzhou Medical University Second Affiliated Hospital

Xiaodong Chen

Wenzhou Medical University Second Affiliated Hospital

\section{Zhiguang Zhao}

Wenzhou Medical University Second Affiliated Hospital

Jian Chen

Wenzhou Medical University Second Affiliated Hospital

Xian Shen ( $\nabla$ shenxian5166@gmail.com )

Wenzhou Medical University Second Affiliated Hospital

\section{Research article}

Keywords: hepatoid gastric adenocarcinoma, gastric cancer, serum alpha-fetoprotein, liver metastasis

Posted Date: November 20th, 2019

DOI: https://doi.org/10.21203/rs.2.17526/v1

License: (c) (1) This work is licensed under a Creative Commons Attribution 4.0 International License. Read Full License 


\section{Abstract}

Background Hepatoid gastric adenocarcinoma (HGAC) is a rare gastric malignancy that exhibits the characteristics of differentiated hepatocellular carcinoma and gastric adenocarcinoma. Most cases of HGAC are also accompanied by an elevated serum alpha-fetoprotein (AFP) concentration. Here, we report a rare case of HGAC involving two primary lesions.

Case presentation A 61-year-old man presented at our institution with the complaint of upper abdominal pain. An examination revealed a significantly elevated serum AFP concentration. Abdominal computed tomography revealed a gastric tumour with enlarged peripheral lymph nodes and a cavernous haemangioma in the right anterior hepatic lobe. The patient underwent distal gastrectomy, and postoperative histopathology revealed two hepatoid gastric adenocarcinomas. Immunohistochemically, the tumours were positive for AFP, hepatocyte and chromogranin A ( $\mathrm{CgA})$, with a Ki67 index $>90 \%$. Following a postoperative diagnosis of HGAC, the patient was treated with a chemotherapy regimen of oxaliplatin combined with capecitabine. At the 6-month follow-up, the patient's serum AFP concentration returned to the normal level. No signs of recurrence were detected.

Conclusions Compared with other gastric cancers, HGAC tends to be more malignant and invasive, with a poor prognosis. These tumours are also prone to liver metastasis,but for which without liver metastasis may have a better prognosis. We hope that our experience with this extremely rare case of HGAC involving two different primary lesions without liver metastasis, as well as our literature review and summary of the etiological mechanism, pathological features, treatment and prognosis, will help improve the diagnosis and treatment of HGAC.

\section{Background}

Hepatoid gastric adenocarcinoma (HGAC), which was first reported by Ishikura in $1985,{ }^{1}$ is a rare malignancy, accounting for only $1 \%$ of all gastric cancers. ${ }^{2,3}$ A differentiated HGAC shares characteristics with both hepatocellular carcinoma (HCC) and gastric adenocarcinoma (GC), and is often accompanied by a high serum alpha-fetoprotein (AFP) concentration. However, AFP is not a definitive biomarker, as some patients with HGAC have normal serum AFP concentrations, while some gastric cancers that are not hepatoid adenocarcinomas can produce AFP. Accordingly, Nagai et al. suggested that HGAC should be diagnosed based on its histological characteristics, irrespective of its capacity to produce AFP. ${ }^{4}$

The diagnosis of HGAC is complicated by its lack of association with unique clinical manifestations. The digestive symptoms of this tumour mainly include upper abdominal pain, abdominal distension and/or black stool. HGAC is mostly diagnosed at a middle or more advanced stage. These tumours are highly invasive and prone to lymph node (LN) and liver metastasis.

According to previous studies, the survival outcomes of patients with HGAC depends on the extent of resection of the primary and liver metastatic lesions. However, no targeted treatment scheme or standard chemotherapy regimen has been determined. The prognosis of HGAC also remains worse than that of 
general gastric cancer even after a successful operation, as demonstrated by Liu et al. who reported1-, 3and 5 -year survival rates of $30 \%, 13 \%$ and $9 \%$, and $96 \%, 61 \%$ and $44 \%$ among patients with and without HGAC, respectively. ${ }^{2}$ Here, we present an extremely rare case of HGAC with two different primary lesions and summarise the etiological mechanism, pathological features, treatment and prognosis of HGAC through a literature review.

\section{Case Presentation}

A 61-year-old man visited a local hospital for gastroscopy, with the complaint of upper abdominal pain of 3 years' duration that had become increasingly frequent and severe. He had used omeprazole for this condition. Gastroscopy revealed two ulcers: a 4-cm-diameter lesion on the gastric antrum and a 2-cmdiameter lesion on the back wall of the gastric angle. Histological analysis of the gastroscopic biopsy specimen determined that both lesions met the criteria of poorly to moderately differentiated adenocarcinoma, upon which the patient was transferred to our hospital for further treatment. Upon admission, we determined that he had a more than 20-year history of gout but no history of liver disease and no notable personal or family medical history. His vital signs were stable, and a physical examination revealed no abnormalities other than mild tenderness on his upper abdomen.

A blood analysis indicated a serum AFP concentration of $1147.00 \mathrm{ng} / \mathrm{mL}$ and carcinoembryonic antigen (CEA) concentration of $14.07 \mathrm{ng} / \mathrm{mL}$. No other abnormalities were observed in the other blood counts, biochemical examinations and coagulation tests. Abdominal contrast-enhanced computed tomography (CT) revealed regional thickening of the gastric wall in the gastric antrum (Figure 1), a gastric cancer with enlarged peripheral LNs, a cavernous haemangioma in the right anterior lobe of the liver, multiple intrahepatic cysts and a cyst in the left kidney. Accordingly, a primary diagnosis of gastric cancer with LN metastasis was made.

The patient underwent laparotomy, during which an adhesion of the upper abdomen and multiple liver nodules of the liver were observed. An approximate ascites volume of $100 \mathrm{ml}$ was removed intraoperatively. Intraoperative ultrasonography revealed a haemangioma and hepatic cyst, but no metastatic nodules in the spleen, small intestine, mesocolon or Douglas fossa. Consequently, a distal gastrectomy with radical D2-lymphadenectomy and Billroth II anastomosis were performed. A gross macroscopic analysis of the resected specimen revealed two lesions located at the gastric angle $(L A)$ and lesser curvature of the pyloric canal (LB), respectively. LA was identified as a local ulcer type (Borrmann II 
type) with an approximate size of $2 \mathrm{~cm} \times 1 \mathrm{~cm}$. This lesion was moderately hard, with no serosal

involvement. LB was identified as an infiltrating ulcer type (Borrmann III type) with an approximate size of $5 \mathrm{~cm} \times 3 \mathrm{~cm}$. This lesion was hard, with subserosal invasion (Figure 2).

A postoperative histopathologic analysis led to the classification of both lesions as HGAC(Figure 3). LA was limited to the mucosa and submucosa, whereas LB was confirmed to have infiltrated the subserosa(Table 1), with invasion of the duodenum, vessels and nerves but not the greater omentum or incisal edge. Eight of $34 \mathrm{LNs}$ were positive, and these were located in the pylorus (2/2) and in the group 3 (1/6), group 5 (1/1), group 6 (1/2), group 7 (1/5) and group 8 LNs (2/3). The TNM stage was $T_{3} N_{3 a} M_{0}$. Via immunohistochemistry, the tumour was determined to be positive for Sal-like protein 4 (SALL4), chromogranin $\mathrm{A}(\mathrm{CgA})$ and $\mathrm{CEA}$, but negative for synaptophysin. The Ki67 proliferation index was $>90 \%$ positive (Figure 4).

Table 1 Characteristics of two tumors

\begin{tabular}{|c|c|c|}
\hline Name & LA & LB \\
\hline Gross type & $\begin{array}{c}\text { Local ulcer type (Borrmann II } \\
\text { type) }\end{array}$ & $\begin{array}{c}\text { Infiltrating ulcer type (Borrmann III } \\
\text { type) }\end{array}$ \\
\hline Location & gastric angle & lesser curvature of the pyloric canal \\
\hline Size & $2 \mathrm{~cm} \times 1 \mathrm{~cm}$ & $5 \mathrm{~cm} \times 3 \mathrm{~cm}$ \\
\hline $\begin{array}{r}|c| \\
\text { Histological } \\
\text { classification }\end{array}$ & \multicolumn{2}{|c|}{ infiltrated the subserosa } \\
\hline Infiltrating depth & limited to the mucosa and & \\
& submucosa & \\
\hline
\end{tabular}


The AFP level decreased to $312.20 \mathrm{ng} / \mathrm{mL}$ (versus $1147 \mathrm{ng} / \mathrm{ml}$ preoperatively), and the CEA level decreased to $6.14 \mathrm{ng} / \mathrm{mL}$ (versus $14.07 \mathrm{ng} / \mathrm{mL}$ preoperatively) 5 days postoperatively. The patient experienced no postoperative complications and was discharged 9 days after the operation. One month postoperatively, he returned to the local hospital for chemotherapy with a combined regimen of oxaliplatin and capecitabine (XELOX regimen). After 6 months of chemotherapy, the patient's AFP and CEA levels continued to decline. A follow-up abdominal CT showed no sign of recurrence.

\section{Discussion And Conclusions}

As noted above, HGAC has no unique clinical manifestations, tends to be diagnosed at a later disease stage and is associated with a high risk of LN and liver metastases. In an analysis of 85 cases of HGAC, Inagawa et al. reported only 11 cases of early gastric cancer. ${ }^{5}$ In that study, the patients were predominantly male (male:female ratio $\sim 2: 1$ ) and middle-aged to elderly, and the primary lesions arose most frequently in the antral part of the stomach $(60.2 \%)$, followed by the body of the stomach and fundus of the cardia and stomach. The patients had an average serum AFP concentration of 51130.1 $\mathrm{ng} / \mathrm{mL}$ (range: $1.0-700,000 \mathrm{ng} / \mathrm{mL}$ ) and an average tumour diameter of $6.5 \mathrm{~cm}$.

A hepatoid adenocarcinoma may originate from the stomach, oesophagus, colon, gallbladder, uterus, bladder, pancreas, ovary or other organs. However, HGAC is the most common manifestation, accounting for up to $83.9 \%$ of hepatoid adenocarcinomas. ${ }^{6}$ Most patients with HGAC have an elevated serum AFP concentration according to Boureille et al., who first reported this parameter as a biomarker of GC in $1970 .{ }^{7}$ Ishikura and colleagues believe that this elevated serum AFP level is associated with the fact that the liver and stomach develop from the embryonic foregut. Therefore, some primary gastric cancer tissues exhibit differentiation toward the liver cell lineage and thus exhibit the intrinsic characteristics of both malignancies, including excess production of AFP. ${ }^{1}$ However, AFP production is only an important characteristic of HGAC but is not a prerequisite for diagnosis, as Ishikura et al. also found that some gastric cancer patients whose lesions shared similar histopathologic features with liver cancer did not exhibit elevated serum AFP concentrations. ${ }^{8}$ Other research findings have suggested that primitive hepatoid cells may exhibit some properties of pluripotent stem cells (e.g., germ cells) during the process of tumorigenesis. Consequently, abnormal differentiation can direct certain cancer cells toward the gastrointestinal or hepatic lineage, leading to increases in the serum AFP concentration consistent with liver cancer. ${ }^{9}$

Hepatic metastasis is a frequent complication of HGAC and is the main cause of death in such cases. The mechanism underlying the hepatic metastasis of HGAC may be related to the $c$-Met oncogene which encodes Met, a hepatocyte growth factor receptor.The rate of $L N$ metastasis has been associated with the tumour invasion depth and presence of $c$-Met amplification, and Met protein is strongly expressed in patients with advanced metastatic disease and those with tumours that have invaded the serous layer. 
These observations suggest that strong Met protein expression induces gastric adenocarcinoma cells to become more similar to liver cancer cells and thus enables the survival of the former in liver tissues.

Currently, pathology is the gold standard for the diagnosis of HGAC. A microscopic examination of tumour tissue reveals the differentiation of gastric cancer cells into hepatoid cells. Histopathologically, HGAC is similar to HCC. In both tumour types, the tumour cells grow, proliferate and invade surrounding tissues, and this process is accompanied by obvious venous infiltration. ${ }^{10,11} \mathrm{~A}$ typical HGAC has unique histopathological features and both hepatoid and adenocarcinoma components. From an ultrastructural perspective, the tumour cells in both differentiated components form intestinal epithelial microvilli, and the tissues are all of gastrointestinal origin. HGAC shares some tumour markers with HCC and exhibits some unique markers. The adenocarcinoma components tend to be positive for a1-ACT and a1-AAT and positive or strongly positive for CEA. The liver cancer components tend to be positive or strongly positive for AFP but weakly positive or negative for CEA. The proteases a1-ACT and a1-AAT not only inhibit the activity of fibrin, but also inhibit the reaction between lectin and normal lymphocytes. These characteristics enhance the invasiveness of HGAC and enable its dissemination to the liver and other organs in the abdominal cavity. ${ }^{12}$ SALL4 expression has been detected in the near-parietal stomach, primordial germ cell tumours, enteroblastocytic adenocarcinoma, yolk sac tumours and HGAC. ${ }^{13}$ Because AFP expression in HGAC is sometimes negative and most tumours usually express glypican-3 (GPC3) and SALL4, GPC3 and SALL4 may be more useful than AFP as clinical biomarkers of HGAC. As SALL4 is not expressed in normal liver tissue or liver cancers, SALL4 expression may also help to distinguish HGAC from $\mathrm{HCC} .{ }^{4,13-15}$ In our case, although the tumour shared morphological features with HCC, the expression of AFP and SALL4 enabled us to make a diagnosis of HGAC.

Currently, no targeted treatment for HGAC is available. Complete surgical resection of the primary gastric cancer lesions and liver metastatic lesions is required to ensure a long survival duration. However, the prognosis associated with HGAC remains worse than that of general gastric adenocarcinoma, even after a successful resection. However, complete surgical excision with subsequent systemic chemotherapy may provide a successful resolution in such cases. Takeyama et al. reported a patient with HGAC and multiple liver metastases who was treated with a combined regimen of paclitaxel and 5 -fluorouracil. The patient achieved a reduction and ultimate disappearance of the metastatic lesion and a return of the serum AFP level to the normal level. ${ }^{16}$ In our patient, a combination of surgery and chemotherapy similarly led to a reduction in the serum AFP level and a lack of disease recurrence.

Most gastric cancers arise as single lesions. In our case, however, we present a rare case of HGAC with two major lesions and no liver metastases. In our case, the TNM stage was $\mathrm{T}_{3} \mathrm{~N}_{3 a} \mathrm{M}_{0}$ and the preoperative AFP concentration was $1147.00 \mathrm{ng} / \mathrm{mL}$. Five days after distal gastrectomy with radical D2-

lymphadenectomy, his AFP level had decreased to $312.20 \mathrm{ng} / \mathrm{mL}$. After 6 months of combined oxaliplatin and capecitabine chemotherapy, the patient's AFP level decreased to $8.5 \mathrm{ng} / \mathrm{mL}$ with no radiologic signs of recurrence. 
In conclusion, $\mathrm{HGAC}$ is associated with a high degree of malignancy and poor prognosis, and no targeted treatment is currently available. HGAC may have a better prognosis for those without liver metastasis. We hope that our observations will improve the general understanding of HGAC and promote the diagnosis and treatment of HGAC by enabling an accumulation of cases. Ultimately, we hope that our findings will contribute to an improved prognosis associated with this disease.

\section{Abbreviations}

HGAC: hepatoid gastric adenocarcinoma

AFP: alpha-fetoprotein

CEA: carcinoembryonic antigen

CgA: chromogranin A

GC: gastric adenocarcinoma

GPC3: glypican-3

HCC: hepatocellular carcinoma

LN: lymph node

SALL4: Sal-like protein 4

\section{Declarations}

\section{Ethics approval and consent to participate}

Not applicable.

\section{Consent for publication:}

Written informed consent was obtained from the patient for publication of this Case Report and any accompanying images. A copy of the written

consent is available for review by the Editor of this journal.

\section{Availability of data and materials}

All data generated or analyzed during this case study are included in this published article. 


\section{Competing interests}

The authors declare that they have no competing interests.

\section{Funding}

No sources of funding were utilized in the preparation of this report.

\section{Authors' contributions}

XS "corresponding author" performed the gastrectomy and ZZ performed the pathological analyses.JC contributed to the conception of this case. $\mathrm{CZ}$ designed the Case Report and drafted the manuscript. XC followed-up the patient. All authors have read and approved the final version of this manuscript.

\section{Acknowledgements}

Not applicable

\section{Author details}

${ }^{1}$ Department of Gastrointestinal Surgery,The Second Affiliated Hospital of Whenzhou Medical University,No.109 West College Road,Whenzhou,Zhejiang Province,People's Republic of China.

${ }^{2}$ Department of Pathology,The Second Affiliated Hospital of Whenzhou Medical University,No.109 West College Road,Whenzhou,Zhejiang Province,People's Republic of China.

\section{References}

1. Ishikura H,Fukasawa $\mathrm{Y}, \mathrm{Og}$ gasawara $\mathrm{K}$,et al. An AFP-producing gastric carcinoma with features of hepatic differentiation. A case report [J]. Cancer, 1985,56 (4)凶 840 -848.

2. Liu $X$, Cheng $Y$, Sheng W, et al. Analysis of clinicopathologic features and prognosticfactors inhepatoid adenocarcinomaof thestomach. AmJ Surg Pathol 2010;34:1465-71.

3. Yoshizawa J, Ishizone S, Ikeyama $\mathrm{M}$, et al. Gastric hepatoid adenocarcinoma resulting in a spontaneous gastric perforation: a case report and review of the literature. BMC Cancer 2017;17:368.

4. Nagai E, Ueyama T, Yao T, Tsuneyoshi M. Hepatoid adenocarcinoma of the stomach. A clinicopathologic and immunohistochemical analysis. Cancer. 1993;72:1827-35.

5. Inagawa S,Shimazaki J,Hori M,et al. Hepatoid adenocarcinoma of the stomach[J]. Gastric Cancer,2001,4(1) \43-52. 
6. Su JS,Chen YT,Wang RC,et al. Clinicopathological characteristics in the differential diagnosis of hepatoid adenocarcinoma\a literature review [J]. World J Gastroentero, 2013 ,19(3)®321-327.

7. Bourreille J, Metayer P, Sauger F, Matray F, Fondimare A. Existence of alpha Feto protein during gastric-origin secondary cancer of the liver. Presse Med.1970;78:1277-8.

8. Ishikura $\mathrm{H}$,Kishimoto $\mathrm{T}$,Andachi $\mathrm{H}$,et al.Gastrointestinal hepatoid adenocarcinoma囚venous

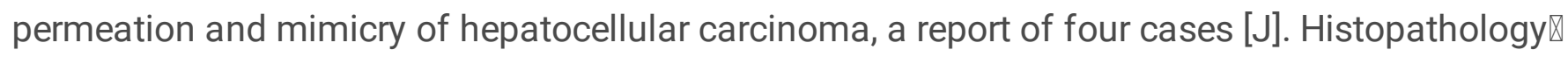
1997ه31(1): 47-54.



10. Kumashiro Y, Yao T, Aishima S, Hirahashi M, Nishiyama K, Yamada T, et al.Hepatoid adenocarcinoma of the stomach: histogenesis and progression in association with intestinal phenotype. Hum Pathol. 2007;38:857-63.

11. Kinjo T, Taniguchi H, Kushima R, Sekine S, Oda I, Saka M, et al. Histologic and immunohistochemical analyses of a-fetoprotein-producing cancer of the stomach. Am J Surg Pathol. 2012;36:56-65.

12. Morinaga S,Takahashi Y. Primary hepatocellular carcinoma and hepatoid adenocarcinoma of the stomach with liver metastasis:an unusual association[J]. Jpn J Clin Oncol, 1996,26(4)『258 -263.

13. Ushiku T, Shinozaki A, Shibahara J, Iwasaki Y, Tateishi Y, Funata N, et al.SALL4 represents fetal gut differentiation of gastric cancer, and is diagnostically useful in distinguishing hepatoid gastric carcinoma from hepatocellular carcinoma. Am J Surg Pathol. 2010;34:533-40.

14. Ushiku T, Uozaki H, Shinozaki A, Ota S, Matsuzaka K, Nomura S, et al. Glypican 3-expressing gastric carcinoma: distinct subgroup unifying hepatoid, clear-cell, and alpha-fetoprotein-producing gastric carcinomas.Cancer Sci. 2009;100:626-32.

15. Sun W, Liu Y, Shou D, Sun Q, Shi J, Chen L, et al. AFP (alpha fetoprotein): who are you in gastrology? Cancer Lett. 2015;357:43-6.

16. Takeyama $H$,Sawai $H$,Wakasugi $T$,et al. Successful paclitaxel-based chemotherapy for an alphafetoprotein-producing gastric cancer patient with multiple liver metastases[J]. World J Surg Oncol $, 2007,5(1) \otimes 1$.

\section{Figures}




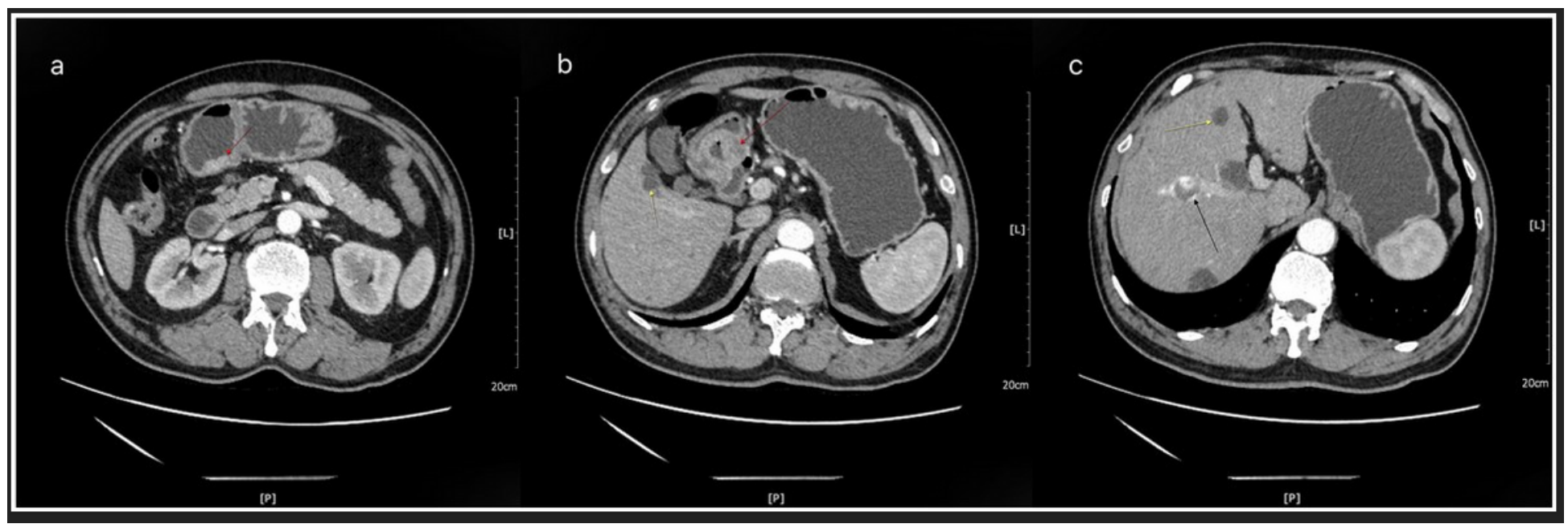

Figure 1

Abdominal contrast-enhanced computed tomography (CT) 


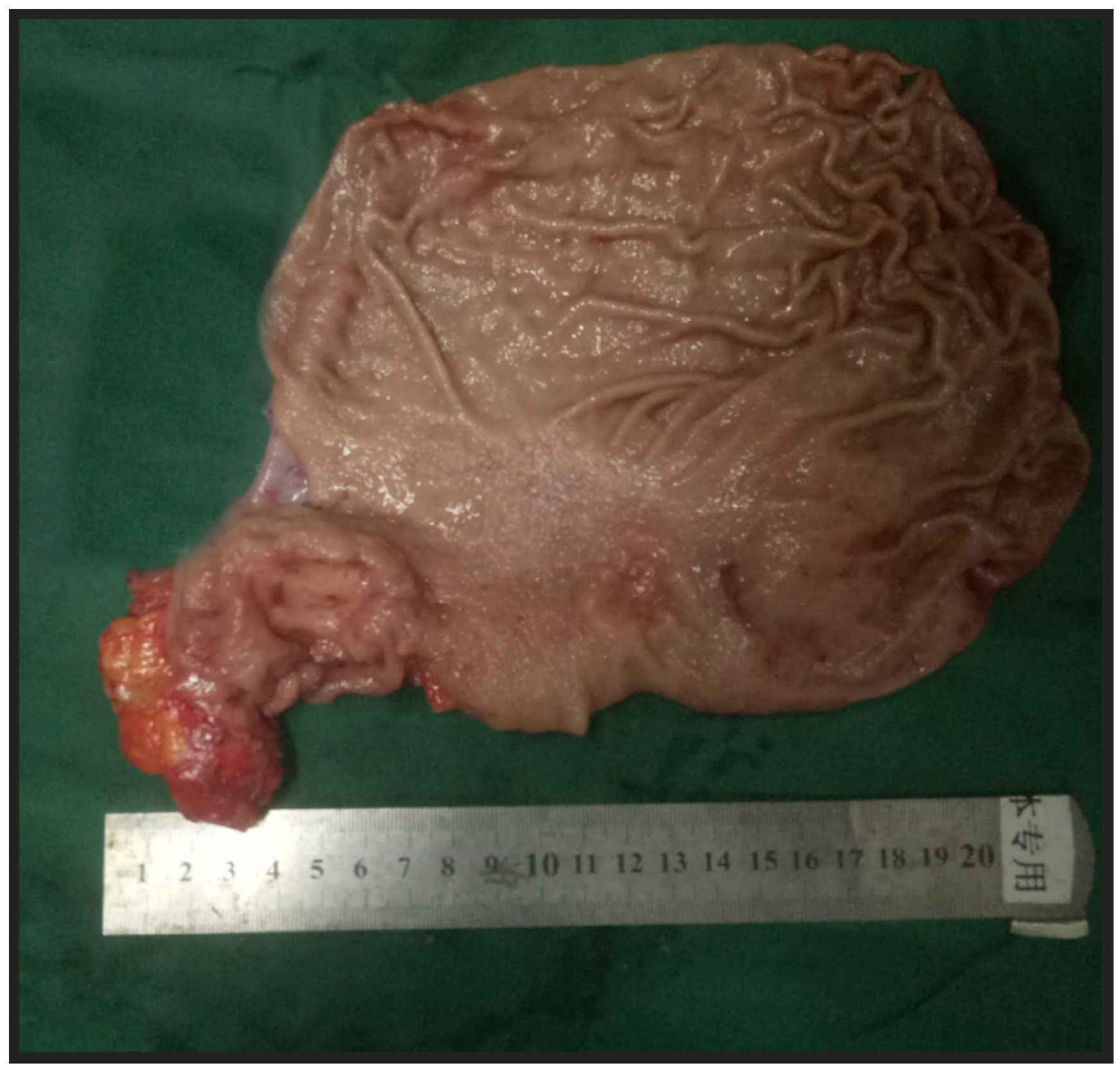

Figure 2

The patient underwent laparotomy, during which an adhesion of the upper abdomen and multiple liver nodules of the liver were observed

2

\section{Figure 3}

A postoperative histopathologic analysis led to the classification of both lesions as HGAC A

\section{Figure 4}


Eight of $34 \mathrm{LNs}$ were positive, and these were located in the pylorus (2/2) and in the group $3(1 / 6)$, group 5 (1/1), group $6(1 / 2)$, group $7(1 / 5)$ and group 8 LNs (2/3). The TNM stage was T3N3aM0. Via immunohistochemistry, the tumour was determined to be positive for Sal-like protein 4 (SALL4), chromogranin $\mathrm{A}(\mathrm{CgA})$ and CEA, but negative for synaptophysin. The Ki67 proliferation index was $>90 \%$ positive.

\section{Supplementary Files}

This is a list of supplementary files associated with this preprint. Click to download.

- CAREchecklist.docx 